\title{
Flux Transfer Events: 1. generation mechanism for strong southward IMF
}

\author{
J. Raeder \\ Space Science Center, University of New Hampshire, Durham, NH 03824, USA
}

Received: 30 December 2004 - Revised: 22 November 2005 - Accepted: 3 January 2006 - Published: 7 March 2006

\begin{abstract}
We use a global numerical model of the interaction of the solar wind and the interplanetary magnetic field with Earth's magnetosphere to study the formation process of Flux Transfer Events (FTEs) during strong southward IMF. We find that: (i) The model produces essentially all observational features expected for FTEs, in particular the bipolar signature of the magnetic field $B_{N}$ component, the correct polarity, duration, and intermittency of that bipolar signature, strong core fields and enhanced core pressure, and flow enhancements; (ii) FTEs only develop for large dipole tilt whereas in the case of no dipole tilt steady magnetic reconnection occurs at the dayside magnetopause; (iii) the basic process by which FTEs are produced is the sequential generation of new X-lines which makes dayside reconnection inherently time dependent and leads to a modified form of dual or multiple X-line reconnection; (iv) the FTE generation process in this model is not dependent on specific assumptions about microscopic processes; (v) the average period of FTEs can be explained by simple geometric arguments involving magnetosheath convection; (vi) FTEs do not develop in the model if the numerical resolution is too coarse leading to too much numerical diffusion; and (vii) FTEs for nearly southward IMF and large dipole tilt, i.e., near solstice, should only develop in the winter hemisphere, which provides a testable prediction of seasonal modulation. The semiannual modulation of intermittent FTE reconnection versus steady reconnection is also expected to modulate magnetospheric and ionospheric convection and may thus contribute to the semiannual variation of geomagnetic activity.
\end{abstract}

Keywords. Magnetospheric physics (Magnetopause, cusp and boundary layers; Magnetospheric configuration and dynamics) - Space plasma physics (Magnetic reconnection)

\section{Introduction}

Magnetic reconnection is the fundamental mode of mass, momentum, and energy transfer from the solar wind and interplanetary magnetic field (IMF) into the magnetosphere-

Correspondence to: J. Raeder

(j.raeder@unh.edu) ionosphere-atmosphere system. Reconnection opens up the magnetosphere so that magnetic field lines of the magnetosphere can directly connect to the IMF. Without reconnection the magnetosphere would be closed and there would be very little influence from the solar wind (SW) and IMF on the magnetosphere.

Although the reconnection between the Earth's magnetic field and the IMF was in dispute at the beginning of magnetospheric research (Axford and Hines, 1961) it is now well established that reconnection occurs at the day side magnetopause. (Haerendel et al., 1978; Paschmann et al., 1979; Cowley, 1980, 1982). However, the in situ signatures of magnetic reconnection at the magnetopause appear to be very different at different times and at different locations. Primarily, one can distinguish between quasi-stationary reconnection (Paschmann et al., 1979) and an apparently timedependent form of reconnection that has been called "Flux Transfer Events" (FTEs) (Russell and Elphic, 1978, 1979).

FTEs are, in the first place, characteristic bipolar signatures of the magnetic field component normal to the magnetopause (Russell and Elphic, 1978; see Elphic (1995) for a recent review of FTE observations and their interpretation). In the original Russell and Elphic (1978) work FTEs were pictured as elbow-shaped flux tubes that originate through a reconnection patch on the magnetopause. Such a flux tube would then accelerate along the magnetopause owing to the $\boldsymbol{j} \times \boldsymbol{B}$ force. Such a flux tube would also exhibit many of the other characteristics of a FTE, for example the existence of magnetospheric and magnetosheath FTEs, the mixture of magnetospheric and magnetosheath plasma usually found within a FTE, and the observed polarity-hemisphere relationship (Rijnbeek et al., 1984; Southwood et al., 1986). However, the main deficiency of this model is that it does not explain why reconnection should happen sporadically, in small patches, and at an average repetition rate of about one FTE every 8 min (Rijnbeek et al., 1984; Lockwood and Wild, 1993). This model has therefore not found universal acceptance. Over the years a number of other models have been proposed to explain FTEs. These models are for the most part of geometrical or phenomenological nature, although some have also been tested using simulations. These models can be broadly categorized into (i) bursty reconnection models 
(Scholer, 1988; Southwood et al., 1986; Ku and Sibeck, $1998,2000)$ where a reconnection burst produces a temporal plasma and field bulge that originates at a single low latitude $\mathrm{X}$-line and propagates to higher latitudes, (ii) multiple $\mathrm{X}$-line models in which several $\mathrm{X}$-lines exist simultaneously at the magnetopause and lead to the formation of plasmoids and flux-ropes (Lee and Fu, 1985, 1986; Fu and Lee, 1985; Shi et al., 1988, 1991; Sonnerup, 1987), or (iii) vorticity induced reconnection in which Kelvin-Helmholtz waves at the magnetopause initiate and control reconnection (Liu and $\mathrm{Fu}$, 1988; Pu et al., 1990). All of these models have in common that they require some form of time-dependent reconnection. However, these models are difficult to test and to distinguish from each other because their predictions are often not precise enough or because the predictions of different models overlap. Even the numerical, three-dimensional models are all local and depend on a number of parameters (boundary conditions, anomalous resistivity) that make them virtually untestable. A concise review and critique of these modeling attempts can be found in Scholer (1995).

Because of the limitations of local models, global modeling of FTEs was attempted using global MHD models of Earth's magnetosphere. Global models have the advantage over local models that they rely less on boundary conditions, although other parameters, for example numerical resistivity, may still affect the results. Early attempts (Sato et al., 1986; Ogino et al., 1989) were too marginal resolved $\left(\sim 0.5 R_{E}\right)$ to yield useful results. More recently, Fedder et al. (2002) have reported results from a global simulation that are roughly consistent with observed FTEs. Specifically, their model produces multiple FTEs consistent with observational evidence (Le et al., 1993). They do not, however, explain why reconnection should be intermittent.

In the following we present FTE results from our own global magnetosphere model. We show that FTEs can be generated when a X-line at the magnetopause does not coincide with the bifurcation of the magnetosheath flow, for example due to dipole tilt. Our model results exhibit several features that have been proposed in previous models but no previous model matches them all. We also show that the FTE development depends on sufficient model resolution and on sufficiently low numerical dissipation. Finally, we show that our model produces spacecraft signatures that are consistent with those that are commonly observed.

\section{Model}

In this study we use the OpenGGCM Geospace General Circulation Model (previously known as the UCLA/NOAA GGCM) that has been used in a number of studies of the solar wind-magnetosphere-ionosphere-thermosphere interaction (Raeder et al., 1998; Raeder, 1999; Raeder et al., 2001a,b; Wang et al., 2003; Raeder, 2003). The model is described in sufficient detail in these papers (we specifically refer to Raeder (2003) for numerical details), thus we only discuss aspects of the model here that are specifically important for this study.

First, the simulation of the FTEs shown in this paper does not require any anomalous resistivity. Adding such an anomalous resistivity term to the otherwise ideal MHD equations has been found necessary in simulations of substorms (Raeder et al., 2001a). However, the anomalous resistivity term does not have any noticeable impact on day side reconnection unless the resistivity threshold (the parameter $\delta$ in the above cited papers) is very low and the normalized resistivity (the parameter $\alpha$ in the above cited papers) is rather high. In the latter case the solutions are comparable to those presented below in Sect. 4 obtained with low numerical resolution. Such behavior is of course expected because low resolution also entails high numerical diffusion. Thus, for FTEs to form in our simulations, the numerical resistivity of the our code is sufficient. The numerical resistivity in the code is a result of the flux-limiting nature of the numerical scheme. Since all finite difference approximations have numerical dispersion they tend to create spurious oscillations at discontinuities, such as at the magnetopause, where the $B_{z}$ field component is discontinuous. These oscillations are prevented by introducing a sufficient amount of numerical diffusion to keep the solution from developing artificial extrema. The OpenGGCM uses the flux-limiting scheme developed by Van Leer (Van Leer, 1973, 1974, 1977) for the induction equation, and a fourth order hybrid scheme for the gasdynamic equations (Harten and Zwas, 1972; Harten, 1983).

Second, the simulations of FTEs presented in this paper have only become possible because increases in computer power have allowed us to run simulations with much improved resolution. Specifically, previous published simulation runs had typically $\sim 10^{6}$ grid cells. Since our numerical grid is somewhat flexible and because it can be adapted to the anticipated solution and to the problem at hand, that number of grid cells usually allowed the resolution in the subsolar region around the bow shock and the magnetopause to be of the order of $0.3-0.4 R_{E}$. The simulations presented in this paper were run with $\sim 8 \times 10^{6}$ grid cells unless otherwise noted. Most of the additional grid cells were packed into the subsolar magnetopause region which allowed a resolution of $0.08 R_{E}(\sim 500 \mathrm{~km})$ in the $\mathrm{X}$-direction and a resolution of $0.2 R_{E}(\sim 1200 \mathrm{~km})$ in the $\mathrm{Y}-$ and Z-directions.

\section{Effect of dipole tilt}

In Figs. 1a and b we compare the results from two simulations which are identical except for the dipole tilt. The solar wind and IMF are kept constant in this simulation at nominal values $\left(N_{\mathrm{SW}}=6.5 \mathrm{~cm}^{-3}, V_{\mathrm{SW}}=450 \mathrm{~km} \mathrm{~s}^{-1}, T_{\mathrm{SW}}=7.7 \mathrm{eV}\right.$, $B_{\mathrm{IMF}}=5 \mathrm{nT}$ ) with due southward IMF. In the simulation with dipole tilt the tilt angle is $34^{\circ}$ which corresponds to the maximum possible tilt angle of Earth that occurs at summer solstice, 21 June, 16:00 UT. The figures show a cut in the noonmidnight meridian $80 \mathrm{~min}$ after the start of the simulation. The color contours represent the plasma pressure, the black 

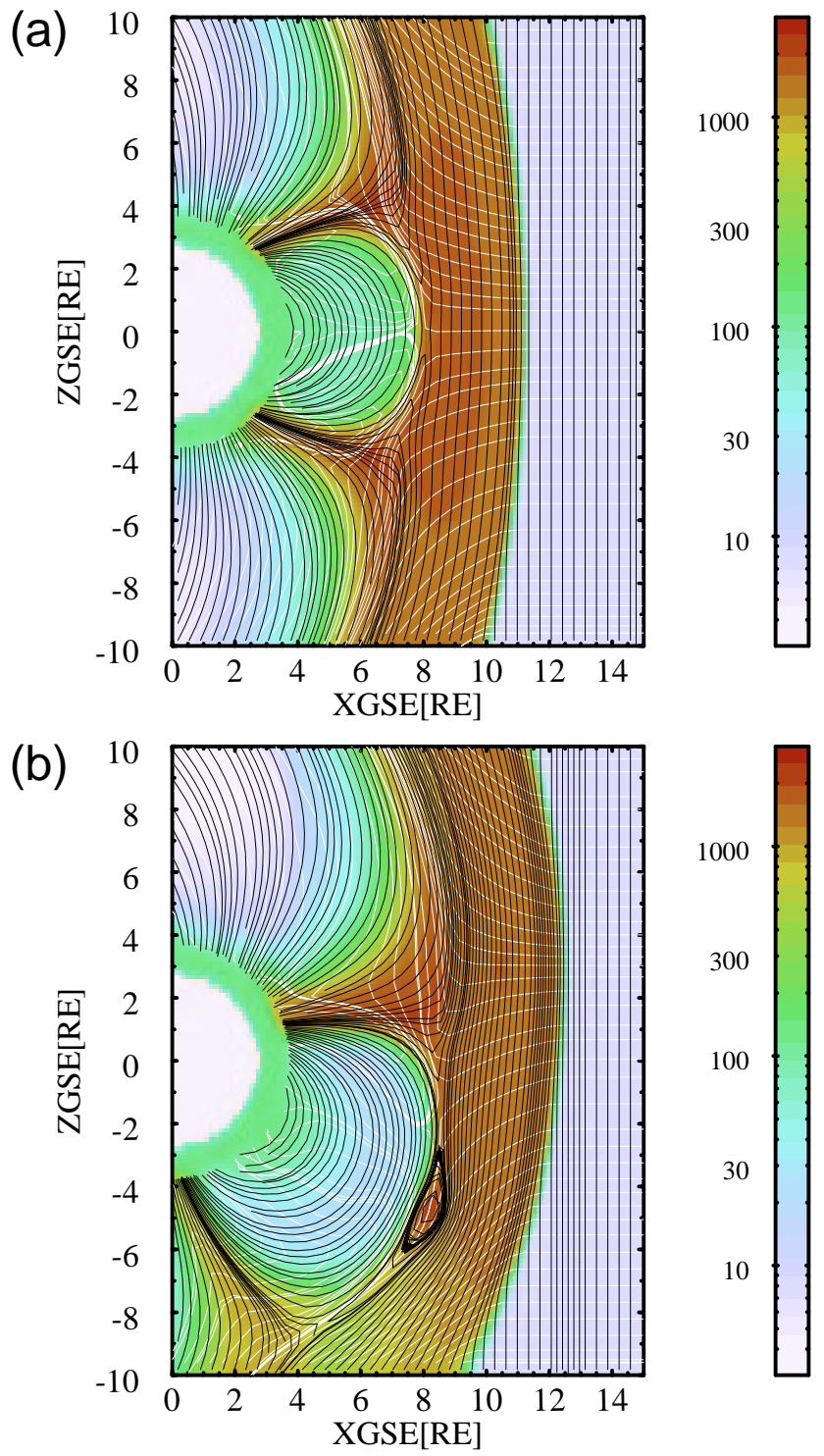

Fig. 1. (a) High-resolution simulation without dipole tilt. There is symmetry between the flow field and the magnetic field, leading to steady reconnection. (b) Same as (a), but with maximum (34 ${ }^{\circ}$ ) dipole tilt. There are multiple X-lines that lead to the formation of FTEs. The color represents the plasma pressure.

lines represent field lines, and the white lines represent flow lines.

Before we discuss the following figures we need to define the term "stagnation flowline". In steady state flow around a sphere, for example, the stagnation flowline is a mathematically singular streamline, i.e., the trajectory of a particle, that comes from infinity and ends on the sphere, where the flow comes to rest. The flow in the simulations shown here is by no means in a steady state, but highly dynamic. We define the stagnation flowline therefore as the flow line that separates magnetosheath flow that turns northward at the magnetopause from flow that turns southward at the magnetopause. This definition is analogous but more general than the definition of the stagnation streamline of the flow around a sphere since the latter also separates flow that goes around the sphere in one way from flow that goes around the sphere in the other way. The main difference is that in our definition the flow does not need to stagnate, and that the line is not necessarily the trajectory of a fluid particle but only tangent to the flow vector everywhere and at any instant in time.

In Fig. 1a (no dipole tilt) the stagnation flowline is parallel to the sun-Earth line and runs directly into the X-line at the magnetopause. This case is strictly symmetric around the sun-Earth line. The flow and the field are steady, except for a some erosion of the day side magnetosphere that lets the magnetopause slowly move inward. This erosion eventually stops when night side reconnection catches up and brings more flux to the day side.

By contrast, Fig. 1b shows an otherwise identical simulation, except for the $34^{\circ}$ dipole tilt towards the sun. In this case plasmoid like structures develop along the magnetopause on the sheath side. Inspection of a series of figures like Fig. 1b shows that these plasmoids form quasi-periodically. There is no strict periodicity and the plasmoids are of different sizes. However their spatial scale does not vary much. The size normal to the magnetopause is of the order of $0.5-1.5 R_{E}$, while the north-south extent is of the order of several $R_{E}$. We will discuss the east-west extent later. Although these structures are not strictly periodic their repetition rate lies between 5 and $15 \mathrm{~min}$.

Clearly, there is no north-south symmetry in the tilted dipole case. The stagnation flowline does not run straight into the magnetopause but is deflected downward. It reaches the magnetopause somewhere between the northern cusp and the nose (as defined by zero magnetic latitude) of the magnetosphere. As we will show later in more detail there is often, but not always, an X-line located where the stagnation flowline reaches the magnetopause. There is also an X-line further south and a plasmoid between these two X-lines. The plasmoid and the latter X-line are embedded in southward magnetosheath flow and thus move southward. As the plasmoid moves southward the field lines that lie northward of the upper X-line are stretched out and dragged southward to form a tongue-like feature extending from the northern cusp southward. Eventually, a new X-line forms closer to the northern cusp that pinches this tongue-like structure off. As a result of the second $\mathrm{X}$-line a new plasmoid structure forms. Within a few minutes this plasmoid convects over the cusp into the tailward magnetosheath. This process then keeps repeating for steady solar wind and IMF conditions. We discuss this process in more detail in Sect. 5 and in Sect. 6 we show that the plasmoid structures have the observational properties expected for FTEs. Thus we will call them FTEs from here on.

An important aspect of the FTE shown here is that its generation does not depend on any external trigger because the solar wind and IMF parameters were held constant in this simulation run. This is consistent with observations (Le et al., 1993) which also show repeated FTEs under constant solar wind and IMF conditions. Of course, that does not 
(a)

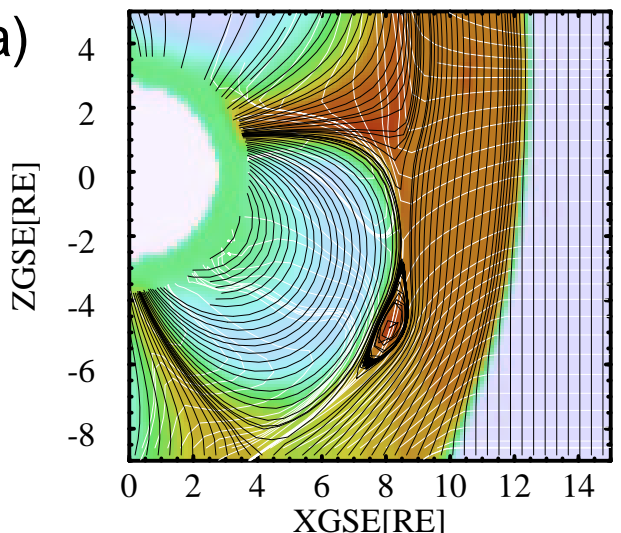

(b)

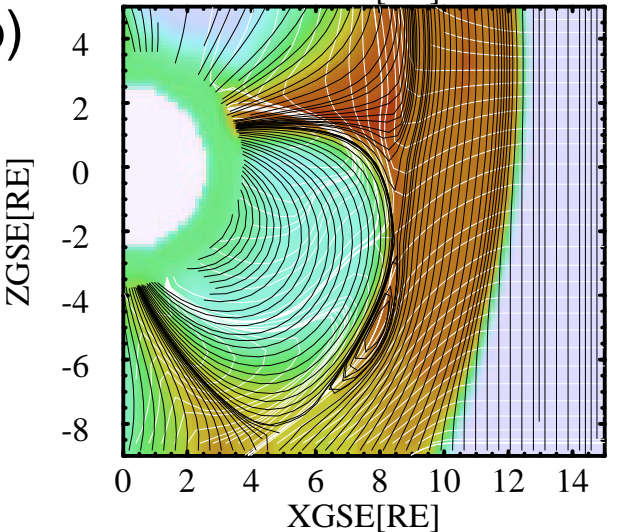

(c)

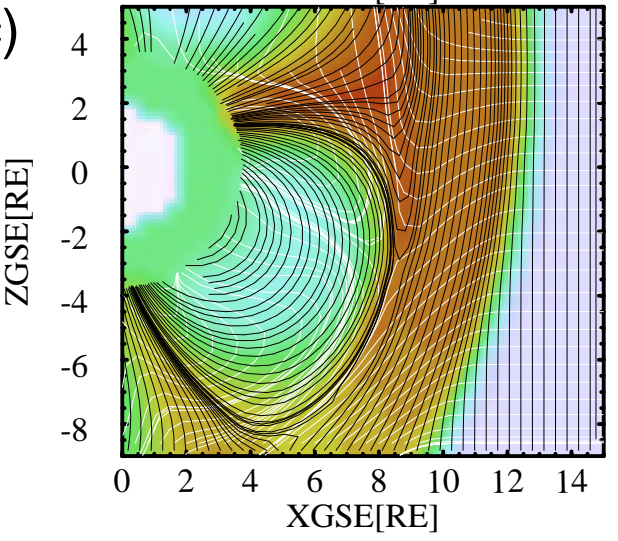

Fig. 2. Three simulations with identical parameters except for the grid resolution: (a) highest resolution $\left(0.08 R_{E}, \sim 500 \mathrm{~km}\right),($ b) medium resolution $\left(0.15 R_{E}, \sim 1000 \mathrm{~km}\right)$, and (c) low resolution $\left(0.3 R_{E}, \sim 2000 \mathrm{~km}\right)$. FTEs only develop fully with the highest resolution. At medium resolution they are smaller and rapidly diffuse away, and at the lowest resolution no FTEs appear but "flow through" reconnection develops instead. The color represents the plasma pressure.

mean that we would not expect for FTEs to form if the solar wind or the IMF were not uniform. We choose the constant input conditions because they make the analysis easier and because it allows us to test the observational constraint that FTEs do not need to be triggered but occur spontaneously. Although reconnection occurs in the model because of numerical resistivity the process shown here does not strongly depend on the numerical resolution or additional resistivity, as long as the resistivity is low enough as to not overwhelm the FTE generation by diffusion. We show in the next section the effect of numerical resolution, and thus numerical dissipation. In the parameter regime studied here the FTEs are only insofar affected as they do not develop if the resolution is too low and thus diffusion is too high. Their other properties, in particular their repetition rate is only weakly affected by the intrinsic resistivity.

\section{Effect of numerical resolution}

Figure 2 shows in the same format as Fig. 1 three simulations with the same setup and the same boundary conditions as in the previous case, except for different numerical resolution. The result shown in panel (a) is from a simulation run with $0.08 R_{E}(\sim 500 \mathrm{~km})$ resolution near the subsolar magnetopause, identical to the one shown above. Panel (b) shows the result from a simulation run with roughly twice the cell size compared to panel (a), i.e., $0.15 R_{E}(\sim 1000 \mathrm{~km})$. In panel (c) the cell size is again increased by a factor of $\sim 2$ to $\sim 0.3 R_{E}(\sim 2000 \mathrm{~km})$. As the cell size increases the FTEs become smaller and eventually disappear. There are two possible reasons for this. First, the FTE could be under-resolved in the coarser simulations. However, the scale of the FTE is at least $1 R_{E}$ in each dimension, thus even simulation run (c) should still resolve the FTE. The second reason is the dependence of the inherent numerical resistivity and diffusion on the cell size. The numerical resistivity $\eta$ in the simulation code is approximately $\eta \sim h^{2}$, where $h$ is the cell size. Thus, the diffusion is about 16 times larger in (c) compared to (a). When the Figs. $2 \mathrm{a}-\mathrm{c}$ are viewed as a fast sequence (movie or animation) one can occasionally see small FTEs develop in run (c), however, they rapidly diffuse away. Thus, the primary reason that FTEs are now within the realm of global simulations is the fact that improved resolution suppresses numerical diffusion sufficiently for FTEs to form and to survive. In the case where the resolution is not sufficient reconnection appears steady, much like the proposed "flowthrough" reconnection (Siscoe et al., 2002).

\section{Generation mechanism}

The FTE generation mechanism has already been partly addressed in Sect. 2. Here we provide further details. Figure 3 shows cuts in the noon-midnight meridian at 4 different times, covering the cycle between the two consecutive FTEs. The FTE seen in Fig. 3a is not the first FTE in this simulation; several FTEs have already occurred before. In Fig. 3 the color coding represents the Z-component of the plasma velocity and the thick green line is the zero contour of that component. Thus, where this contour intersects the magnetopause is also the stagnation point, using the definition of the stagnation point that we introduced before. This can also be seen in the flow lines (red), which have a triple-point (three lines converging) very close to that location. The black lines are field lines. 
(a)

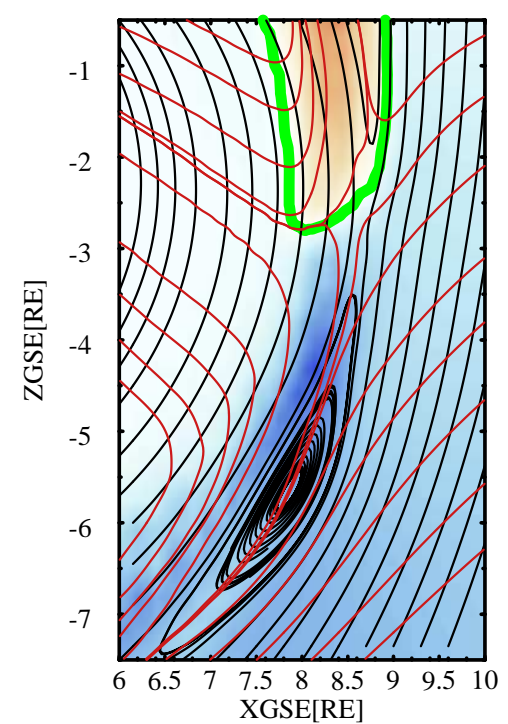

(c)

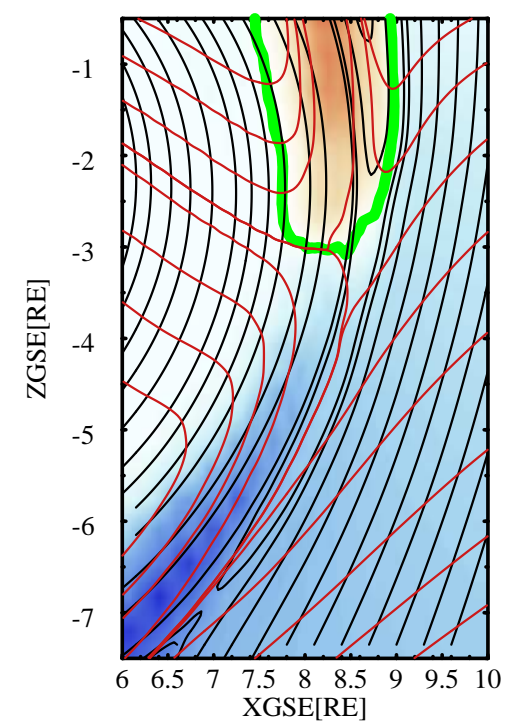

(b)
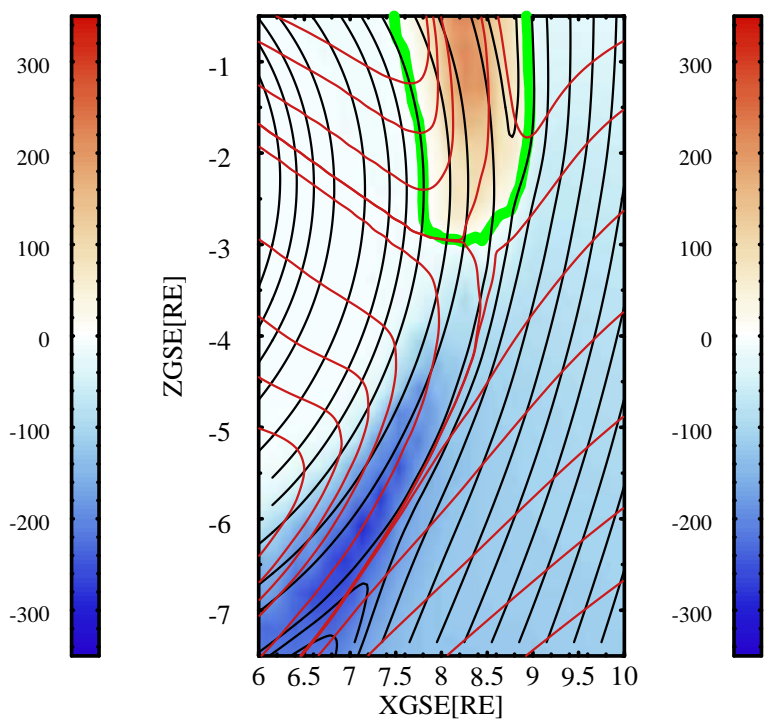

(d)

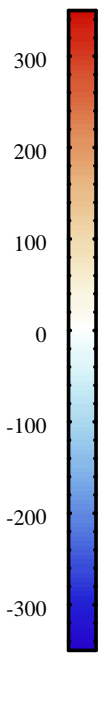

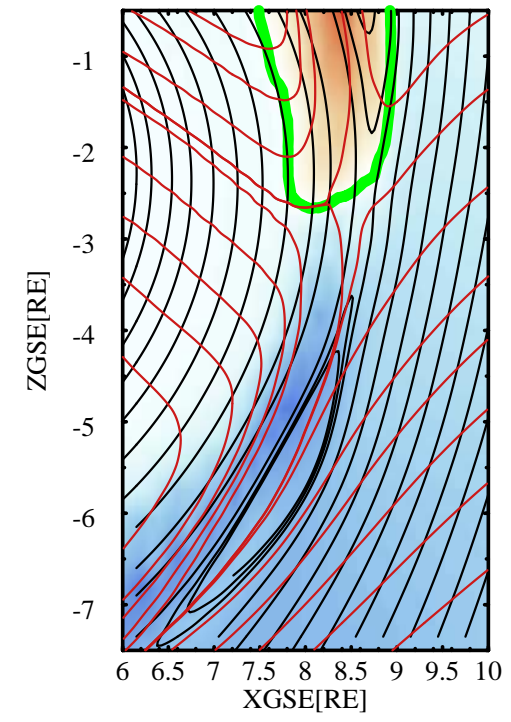

Fig. 3. The four panels show cuts in the noon-midnight meridional plane at different times. In each of the panels the color coding represents the Z-component of the plasma velocity in $\mathrm{km} / \mathrm{s}$. The thick green line is the zero contour of the plasma velocity Z-component. The black lines are field lines, i.e., these lines are tangent to the magnetic field direction in the $Y=0$ plane. The red lines are flow lines, i.e., these lines are tangent to the instantaneous velocity direction in the $\mathrm{Y}=0$ plane. These snapshots were taken at different times: $(\mathbf{a}) \mathrm{t}=\mathrm{t}_{0}=3260 \mathrm{~s},(\mathbf{b})$ $\mathrm{t}=3360 \mathrm{~s}=\mathrm{t}_{0}+100 \mathrm{~s},(\mathbf{c}) \mathrm{t}=3400 \mathrm{~s}=\mathrm{t}_{0}+140 \mathrm{~s},(\mathbf{d}) \mathrm{t}=3610 \mathrm{~s}=\mathrm{t}_{0}+350 \mathrm{~s}$.

At the beginning of the sequence (Fig. 3a) a FTE has developed. This FTE is bordered by two X-points. One X-point is located at the lower end of the figure, and the second one is located where the stagnation flowline impinges upon the magnetopause. The entire FTE is located in southward flow from the magnetosheath, which is enhanced by outflow from the upper X-point and somewhat decelerated by the outflow from the lower X-point.

In Fig. 3b, $100 \mathrm{~s}$ later, the plasmoid has moved southward. The upper edge of the FTE has moved from $\mathrm{Z} \sim-3.5 R_{E}$ to $\mathrm{Z} \sim-6.5 R_{E}$, i.e, $3 R_{E}$ in $100 \mathrm{~s}$, or $\sim 180 \mathrm{~km} / \mathrm{s}$, consistent with the flow velocity. There is no upper X-point to make out in Fig. 3b. Instead, at this time there is a long thin current sheet that runs between the point in the north where the $\mathrm{X}$ point used to be and its southern end which is defined by the northern tip of the departing FTE. In terms of magnetic topology these two ends of the current sheet are Y-points. Thus, what used to be an X-point has now been stretched into two Y-points connected by a current sheet.

However, Fig. 3c, the snapshot $40 \mathrm{~s}$ after the one shown in Fig. 3b, shows that the Y-point associated with the departing plasmoid, now lying at $(\mathrm{X}, \mathrm{Z}) \sim(7,-7) R_{E}$, is becoming an 

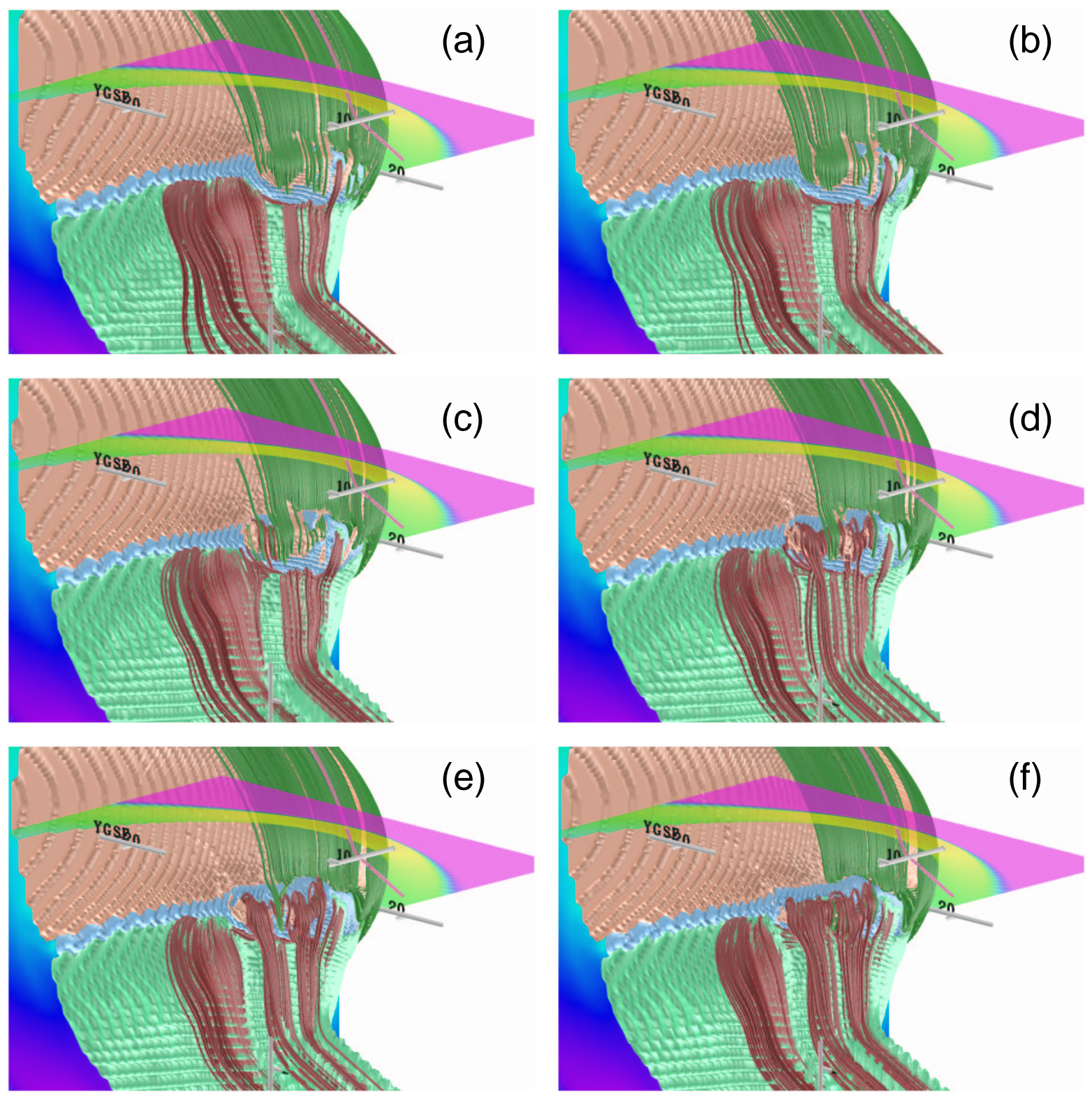

Fig. 4. Three-dimensional perspective view of FTE generation. The panels are snapshots taken every $12 \mathrm{~s}$. The view is from the south, dawn, and sunward side of the magnetosphere, i.e., the sun is to the right. The plane at $\mathrm{z}=3 R_{E}$ is color coded with the plasma pressure. The 3 surfaces are magnetic separators, the green field lines are anchored in the Northern Hemisphere, and the red field lines are anchored in the Southern Hemisphere. The blue bands of the closed field line separator surface that are visible essentially mark the location of the X-lines present at the dayside magnetopause. See text for more details.

X-point again. In other words, the current sheet tears exactly at the Y-point to form a new X-point. However, there is apparently no change at the northern end of the current sheet where the Y-point persists. As a result, there is a tongue of magnetic flux extending from the Y-point to the $\mathrm{X}$-point.

Figure $3 \mathrm{~d}, 350 \mathrm{~s}$ after the beginning of this sequence, and $210 \mathrm{~s}$ after the Fig. 3c snapshot now shows that a new X-point has formed where the northern Y-point had been and where the stagnation flowline impinges on the magnetopause. The formation of this X-point may be understood by the fact that the flows converge here on the current sheet. Between the southern X-point, which has moved further south, and this new X-point there is now a new FTE. This FTE has not yet reached the same size as the one in Fig. 3a. Inspection of subsequent snapshots shows that it will grow for another $\sim 120 \mathrm{~s}$ until a stage very similar to the one in Fig. $3 \mathrm{a}$ is reached and the cycle repeats.

Figure 4 shows how the FTE generation process works in three dimensions. The parameters for this run are the same as for the highest resolution run shown in Figs. 2 and 3, except that for this run there was a small IMF $B_{y}$ component such that the IMF clock angle is $165^{\circ}$, i.e., $15^{\circ}$ off the 
north-south direction towards dusk. The small IMF $B_{y}$ component breaks the East-West symmetry and is probably more realistic considering the fact that the IMF is never due south for an extended period. The FTE formation process is not affected by the small IMF $B_{y}$ and still looks the same as previously shown for the due south IMF case. However, the IMF $B_{y}$ causes the FTEs to have an axial field component, which makes them flux-ropes.

These renderings of the magnetospheric plasma and field configuration consist of several elements. The view is from the south and dawn, such that the sun is to the right. The plane given by $\mathrm{z}=3 R_{E}$ is color-coded with the plasma pressure. The bow shock is marked by the transition from magenta to yellow and green, showing the jump in plasma pressure across the shock. The pink, green, and blue rippled surfaces are magnetic separators. The pink surface envelopes the lobe flux that is anchored in the Northern Hemisphere, the green surface envelopes the lobe flux that is anchored in the Southern Hemisphere, and the blue surface delineates the outer boundary of closed magnetic flux. The surfaces appear rippled because of the finite grid size. The smaller ripple size near the center reflects the higher numerical resolution there. Ideally, the pink and green surfaces should touch each other, and the line along which they touch would be the magnetic X-line where magnetic reconnection occurs. However, we have slightly displaced these surfaces so that a small gap between them remains through which the blue surface is visible. If reconnection were steady, this blue band would simply run across the dayside. However, as the figure shows, the situation is more complicated. In addition to the flux surfaces there are also a number of selected field lines drawn. The red lines connect to the Southern Hemisphere and the green lines connect to the Northern Hemisphere. There is no relation between the field lines shown in different panels. Although the figure suggests that the lines are moving, the lines in each panel are different and merely drawn to better visualize the field topology.

The panels (a) through (f) of Fig. 4 are snapshots in time taken $12 \mathrm{~s}$ apart. The whole sequence thus covers $60 \mathrm{~s}$, i.e, $1 \mathrm{~min}$. At the beginning in panel (a) there appears to be just one $\mathrm{X}$-line across the dayside magnetosphere, which makes a southward excursion near local noon. However, to the north of that X-line, near the subsolar point, there is already a new $\mathrm{X}$-line forming, but it is still hidden behind the tongue of flux that drapes southward over the subsolar region. Within the next $\sim 30$ s (panels (b), (c), and (d)) the reconnection process associated with this $\mathrm{X}$-line eats its way through this overhanging flux, so that a new $X$-line appears. At this time, well visible in panel (e), there exist 2 simultaneous X-lines near the subsolar point on the magnetopause, separated by $\sim 3-5 R_{E}$ in the north-south direction. The width, i.e., the east-west extent, of this dual X-line region is $\sim 15-20 R_{E}$. The magnetic field that became severed from the overhanging flux has a flux-rope like structure. This is in part due to the $B_{y}$ component of the IMF, and also in part due to the draping of the IMF over the magnetopause which creates a $B_{y}$ field component even in the case of pure southward
IMF. As time progresses the flux-rope, which is south of the stagnation stream line, is dragged southward by the magnetosheath flow, and eventually moves over the southern cusp. As that happens, a new flux-rope is already forming further to the dusk side, which is barely visible through its overhanging green flux tubes.

An important implication of this process is its seasonal dependency. The dipole orientation of this simulation is at its maximum Northern Hemisphere summer value. The fluxropes that are generated at the magnetopause all move in the southern direction, and, as we will show later, there are no indications of flux-ropes in the Northern Hemisphere. Because of the symmetry one can generalize this statement somewhat to include the northern winter (southern summer) season, assuming that the flux-ropes seen in Fig. 4 are indeed FTEs: If the IMF is strongly southward $\left(B_{z} \gg B_{y, x}\right)$ and near solstice, FTEs should only occur in the winter hemisphere, while the reconnection flows at the magnetopause in the summer hemisphere are steady. This prediction is statistically testable if enough observations are available that fulfill these criteria and if orbital bias is taken into account. One should note, however, that this prediction says nothing about the FTE generation near the equinoxes or when a strong IMF $B_{y}$ is present.

\section{Spacecraft signature}

It remains to be shown that the flux-ropes in Fig. 3 are indeed FTEs. The defining characteristic of FTEs is their bipolar signature in the magnetic field normal component $B_{N}$ when observed by a spacecraft, and possible other signatures in other variables (see, e.g., Elphic, 1995). In order to test for these signatures we have produced time series of these variables at several locations near the magnetopause.

Figure 5 shows time series taken in the noon-midnight meridian at a geocentric distance of $9.8 R_{E}$ and at $-30^{\circ} \mathrm{GSE}$ latitude. This location is near the nose of the magnetosphere. We estimate that the normal to the magnetopause at this location points $14^{\circ}$ south in GSE latitude, i.e., $N=(0.97,0.0$, $-0.24)$ in GSE coordinates. The magnetic field is transformed into the usual boundary coordinates (Russell and Elphic, 1978), where $\boldsymbol{N}$ points normal and outward from the magnetopause, $\boldsymbol{L}$ points roughly northward such that it is perpendicular to $\boldsymbol{N}$ and that the plane spanned by $\boldsymbol{L}$ and $\boldsymbol{N}$ contains the dipole axis, and $\boldsymbol{M}$ completes the right-handed orthogonal coordinate system and points roughly westward. The top panel shows the L, M, and M components of the magnetic field, followed by the magnetic field magnitude, the plasma pressure, the plasma density, and the plasma bulk velocity. The time axis starts 00:30 h after the start of the simulation until 01:35 h into the simulation. The simulation result agrees with virtually all expected FTE signatures:

- There are clear bipolar signatures in the magnetic field $B_{N}$ component at 00:36, 00:45, 00:57, 01:04, 01:20, 01:26, and 01:32. Each of these signatures 


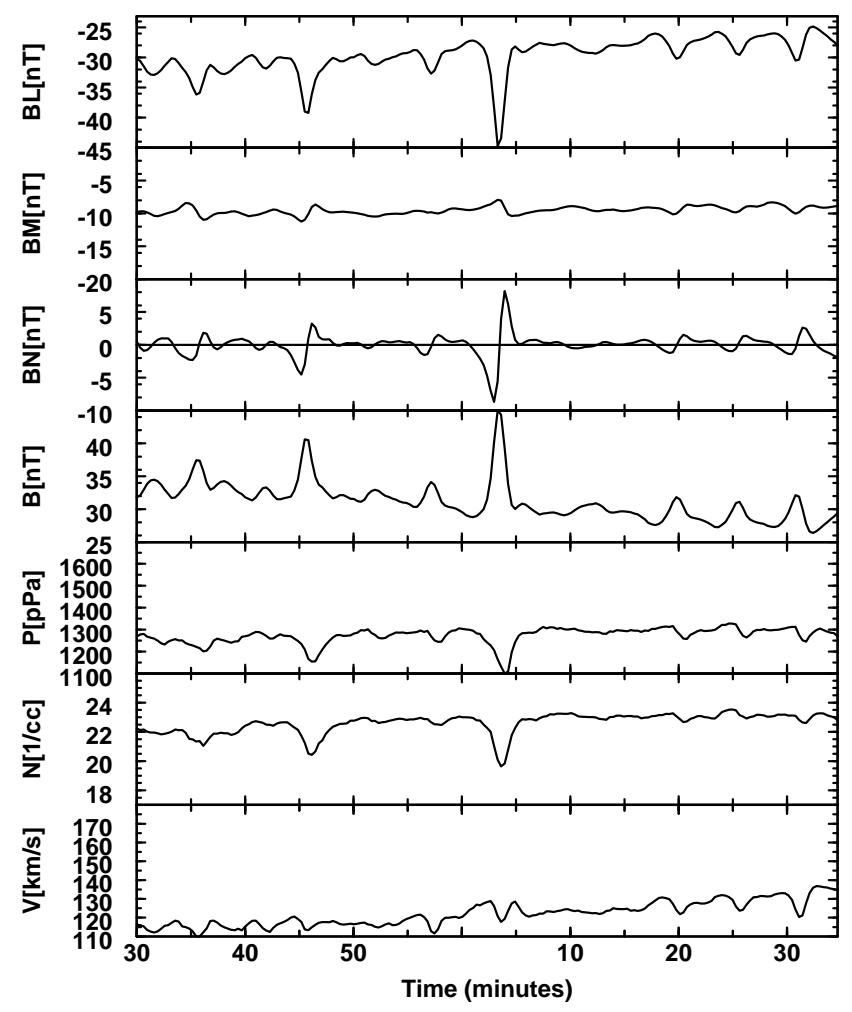

Fig. 5. Time traces of a virtual satellite taken at $9.8 R_{E}$ geocentric distance and $-30^{\circ}$ GSE latitude. This location is roughly at the center of the magnetosphere, in the magnetosheath, and close to the magnetopause. The panels show, from top to bottom, the L, M, and $\mathrm{N}$ components of the magnetic field (see text for details), the total field, the plasma pressure, the plasma density, and the plasma velocity. There are clear dipolar signatures in the magnetic field $\mathrm{N}$ component at $35,57,04,20,26$, and $32 \mathrm{~min}$.

corresponds to a flux-rope moving across the virtual satellite location.

- The polarity of the bipolar signature is of the "reverse" $(-/+)$ kind (Rijnbeek et al., 1982, 1984; Berchem and Russell, 1984; Southwood et al., 1986; Kawano et al., 1992), which is consistent with FTEs in the Southern Hemisphere.

- The amplitude of the bipolar signature varies between $5 \mathrm{nT}$ and $30 \mathrm{nT}$ peak-to-peak which is well within the range of typical observed FTEs.

- The duration of the bipolar signatures is $2-4 \mathrm{~min}$, which is somewhat larger than typically observed for FTEs (Saunders et al., 1984), but still within range (for example, the paper by Russell and Elphic (1979) shows a 4-min FTE). However, the determination of the duration is somewhat subjective since the beginning and end of the bipolar structure is difficult to determine. Because the grid resolution is still not ideal one would also expect that the flux-ropes tend to smear out in the

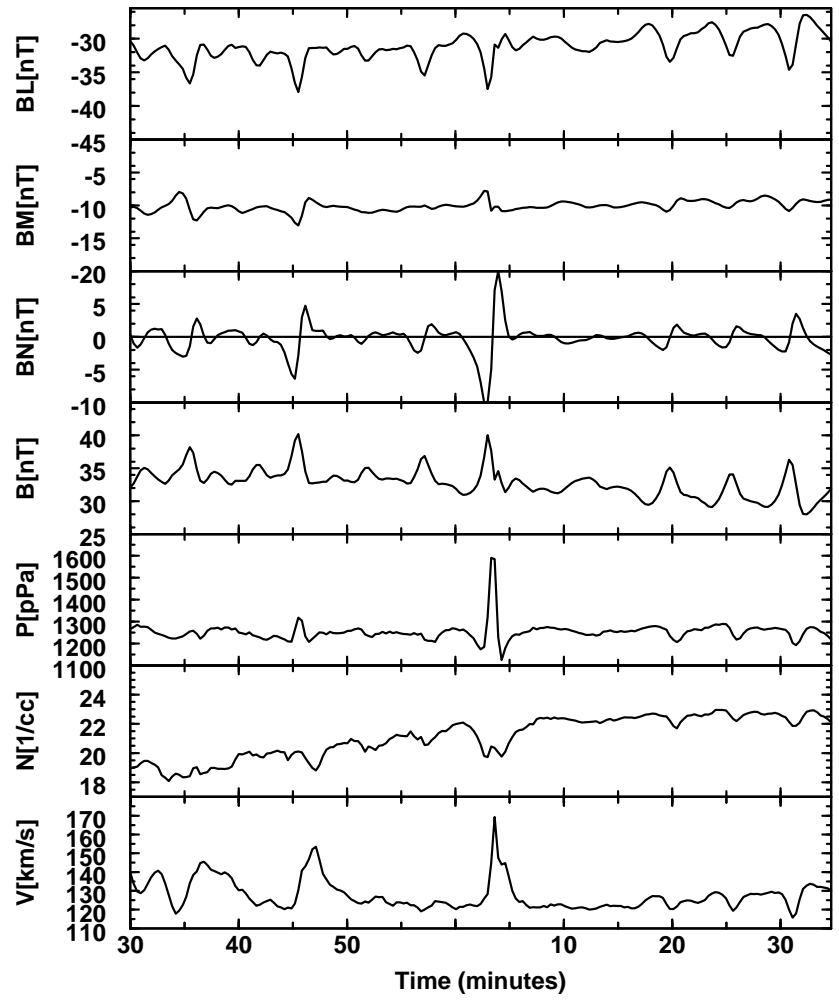

Fig. 6. Same as Fig. 4, except that the traces are taken $0.2 R_{E}$ further outward, at $9.8 R_{E}$.

simulation, which increases the duration of the bipolar signature.

- The bipolar signatures in the simulation occur mostly non-periodic. The average repetition rate is somewhat less than $10 \mathrm{~min}$ (7 FTEs in $65 \mathrm{~min}$ ). The time between the signatures ranges from $6 \mathrm{~min}$ to $16 \mathrm{~min}$, which is consistent with observation statistics (Rijnbeek et al., 1984) that show an average cadence of 8 min with a broad range of inter-FTE durations.

- The bipolar signatures in the simulation occur during negative $B_{L}$, thus in the magnetosheath. They correspond to an enhanced core field, which is mainly caused by an enhancement of the $B_{L}$ component. This characteristic is consistent with a B or C type FTE in Elphic's (1995) taxonomy.

- The bipolar signatures in the simulation and the enhanced core field correspond to density and pressure decreases. This is also consistent with a B or C type FTE, which show both a small density and temperature enhancement (see Fig. 5a in Elphic, 1995) and in Paschmann et al. (1982).

- The bipolar signatures in Fig. 5 show only small and non-systematic variations in the bulk flow velocity. However, when the virtual satellite is moved $0.2 R_{E}$ 
inward (Fig. 6) there are significant flow velocity enhancements that correspond to some of the bipolar $B_{N}$ signatures. This is also consistent with B/C type FTEs which sometimes, but not always have flow velocity increases (Paschmann et al., 1982; Klumpar et al., 1990; Elphic, 1995).

- While the bipolar signatures in Fig. 5 correspond to pressure dips, this is no longer the case in Fig. 6, where 2 of the bipolar signatures have both pressure and magnetic field increases. Thus, these structures are apparently not in simple pressure equilibrium in the sense that the sum of plasma and magnetic pressure are not constant, but with enhanced core pressure, as often observed in FTEs (Paschmann et al., 1982; Farrugia et al., 1988). Of course, the FTEs still obey $\nabla p=\boldsymbol{j} \times \boldsymbol{B}$ to a very good approximation. The enhanced core pressure is balanced by the curvature tension of the magnetic field.

- The bipolar signatures occur spontaneous during southward IMF, without any trigger, consistent with observations (Le et al., 1993).

The simulation thus produces essentially all characteristics of FTEs that can be expected from a MHD model. Energetic particles and plasma distribution functions are also often used to characterize FTEs (Thomsen et al., 1987) but they can of course not be reproduced by this model. FTEs also have structure at smaller scales (Farrugia et al., 1988). Resolving such small structures is currently beyond the capabilities of our model but may be possible in the future with better resolved simulations because these structures are still in the MHD regime. Nonetheless, the consistency of the bipolar structures seen in our simulation lets us conclude with confidence that they are indeed the same as FTEs.

\section{Discussion}

Flux-ropes have been produced in prior global simulations, for example with the LFM code (Fedder et al., 2002) and with the BATS 'R'US code (M. Kuznetsova, personal communication). In the case of the Fedder et al. (2002) paper it has been shown that the expected spacecraft signatures are similar to those of FTEs. That work, however, differs significantly from ours in that the simulation has no dipole tilt but a stronger IMF $B_{y}$ component. Fedder et al. (2002) interpret their results as supporting a single $\mathrm{X}$-line bursty reconnection process as proposed previously by several authors (Scholer, 1988, 1989; Ku and Sibeck, 1998; Scholer, 1995), modified by 3 -D effects. They give no explanation as to why the reconnection process is bursty or why the repetition rate of FTEs in their simulation is of the order of $8 \mathrm{~min}$.

In the simulation results shown above the basic process clearly involves dual X-lines. A dual (or multiple) X-line (MXR) process has been considered previously by several authors (Lee and Fu, 1985, 1986; Fu and Lee, 1985; Shi et al.,
1988, 1991; Sonnerup, 1987). These multiple X-line reconnection (MXR) models are mostly phenomenological or 2-D simulations in which multiple X-lines are forced to occur, for example, by locally enhanced resistivity. They generally do not explain why several X-lines should occur simultaneously but focus on the consequences. The results presented in this paper show that once two X-lines have formed the reconnection process can no longer be stationary. The flow around the magnetopause must bifurcate, and it does so at one of the X-lines. That leaves the other X-line and the O-line in between inevitably in a flow that is directed away from the first X-line. As the second X-line and the O-line are convected away from the first $\mathrm{X}$-line the first $\mathrm{X}$-line becomes elongated into a thin current sheet. This current sheet then forms a new X-line where the stagnation flow line impinges on the magnetopause, thereby repeating the cycle. The main difference between our model and other MXR models is that in our model the X-lines do not form simultaneously but sequentially.

In our discussion of the generation mechanism we have only considered the situation where a new FTE forms after one that already existed. This begs the question how the first FTE forms. In the simulations shown here the first FTE formed as a result of the startup process, where the solar wind with a southward IMF is blown onto a dipole. The magnetopause first forms as a thin current layer which subsequently tears and forms the first FTE in that manner. This also happens in the case of no dipole tilt, where two islands form simultaneously and convect away from the subsolar point. However, in the case of no dipole tilt, after these initial islands have convected away only a single X-line remains at the equator and reconnection proceeds is a steady fashion.

From the reconnection geometry and from the typical plasma parameters in the magnetosheath we can derive the average FTE repetition rate. From Fig. $1 \mathrm{~b}$ we estimate the length of the FTE along the magnetopause, from X-line to Xline (i.e., from where the stagnation stream line touches the magnetopause to the southern cusp), to be $l \sim 8-10 R_{E}$. The appearance of the next FTE should then be determined by the convection time of the existing FTE over its entire length in the direction of convection, i.e., downward. This velocity is essentially given by half the magnetosheath flow speed of $\sim 200 \mathrm{~km} / \mathrm{s}$, i.e., $v \sim 100 \mathrm{~km} / \mathrm{s}$, because the FTE needs to accelerate from zero velocity near the stagnation streamline to its full magnetosheath velocity. The repetition time is thus estimated to be of the order of $l / v \sim 8-10 \mathrm{~min}$, which is what we see in the simulation and what is typically observed. Such a simplified model would predict, however, strict periodicity of the FTE occurrence if the solar wind and IMF conditions are steady. This is not what the model shows. The discrepancy can be explained by inspection of Fig. 4. Apparently, there is no east-west symmetry, which the simple model assumes, but the FTEs have limited spatial extent in the East-West direction. FTEs occur thus at different local times and it is not too far-fetched to assume that the dynamics of one FTE will affect the development of the next one. 


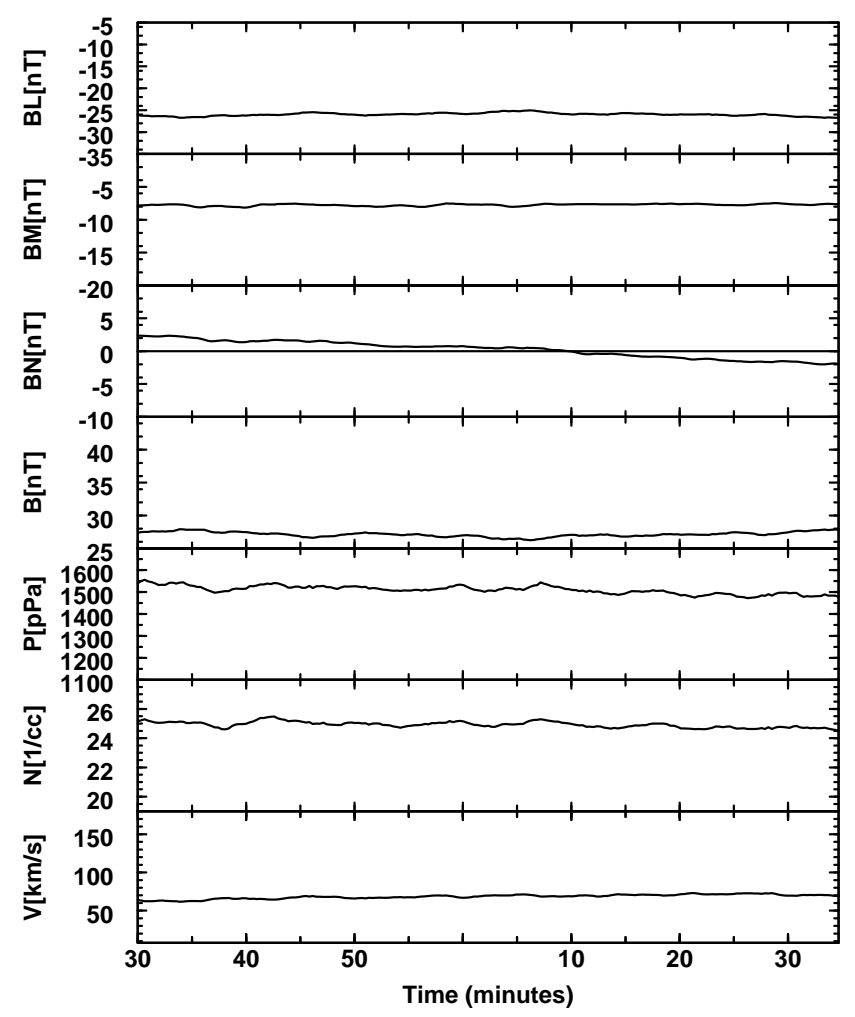

Fig. 7. Same as Fig. 5, except that the traces are taken in the summer hemisphere at $0^{\circ}$ GSE latitude. There are no indications of FTEs.

The system as a whole is thus sufficiently non-linear to allow for quasi-periodicity, but not for strict periodicity. This situation is similar to the dynamics of two coupled pendulums, which despite being a comparatively simple system, produces chaotic behavior.

As noted above, the FTE generation process has a strong seasonal dependence. To strengthen this point, Figure 7 shows time series from a virtual satellite in the summer hemisphere. There are no variations, whatsoever, that could be identified as FTEs.

Even if the FTE generation is substantially different for large IMF $B_{y}$ and/or $B_{x}$ the occurrence rate of FTEs should still be modulated by season and hemisphere. Such modulation should be testable from the analysis of FTE statistics, in particular with Cluster II data. Care should be taken, however, to eliminate seasonal bias entering from the orbital parameters.

Although not addressed in detail in this paper, FTEs versus steady reconnection should produce different reconnection rates for the same solar wind and IMF parameters depending on season. Consequently, there would be a modulation of magnetospheric convection and thus a modulation of geomagnetic activity. The seasonal and diurnal modulation of geomagnetic activity is a well known effect (Russell and McPherron, 1973; Cliver et al., 2000; Hakkinen et al., 2003) and usually attributed to three effects: Changes in heliographic latitude during the year; variations of the solar wind flow direction with respect to the dipole axis; and variation of the angle between the GSM equatorial plane and the solar equatorial plane (Russell-McPherron effect) which would favor dayside reconnection for a Parker spiral IMF orientation during the solstices (see Hakkinen et al. (2003) for an overview of the effects). However, neither effect can account for the entire observed variation in geomagnetic activity. The modulation of the reconnection rate by the dipole tilt would produce an additional semiannual variation of geomagnetic activity with a positive phase relationship, i.e., stronger activity during the solstices. However, this effect may be difficult to differentiate from the other hypotheses, in particular from the Russell-McPherron effect.

\section{Summary and conclusions}

We have used high-resolution global simulations of the magnetosphere to investigate the generation mechanism of FTEs during strongly southward IMF with $\left|B_{y, x}\right| \ll\left|B_{z}\right|$. We find that the successful simulation of FTEs depends on the numerical resolution of the model, but that with sufficiently well resolved simulations it is possible to investigate the formation of FTEs. In summary, our key findings are:

1. Under suitable conditions the model produces quasiperiodically flux-ropes at the dayside magnetosphere that have the observational characteristics of FTEs.

2. FTEs are generated by multiple $X$-line reconnection where new X-lines form sequentially.

3. FTE formation is seasonally modulated with no FTE generation (steady reconnection instead) during equinox and exclusively time dependent reconnection with FTE formation during solstices. The seasonal modulation of FTE generation may contribute to the semiannual variation of geomagnetic activity.

4. When FTEs form during periods of large dipole tilt they will only occur in the winter hemisphere. This prediction is testable and allows to distinguish from other models.

5. The model explains the $\sim 8$ min quasi-periodicity by the convection and re-formation time of flux-ropes at the magnetopause.

Although these findings mark a significant step in our understanding of FTE generation, important questions remain: (i) Is the mechanism dependent on the type of resistivity or other kinetic effects that break the frozen-in condition and makes reconnection happen in the first place? (ii) How does the generation mechanism change for larger IMF $B_{y}$ values? Is a large dipole tilt still necessary or could a strong IMF $B_{y}$ also lead to FTE formation. (iii) How will a further increase of the numerical resolution affect the results? Although we strongly believe that the results presented here will still hold if the numerical resolution of the simulations is substantially 
increased, other processes may become "visible", for example the formation of small islands. (iv) How can these results be reconciled with Elphic's (1995) FTE taxonomy? Figures 5 and 6 indicate that FTEs look quite differently depending on the location of the observing spacecraft, but much needs to be done to produce a complete picture, in particular about the fringe effects of FTEs. Many FTEs may go unidentified in data because they do not show the "classical" bipolar $B_{N}$ signatures. (v) What is the fate of FTEs as they propagate over the cusp and into the tail? Evidently, they were first observed near the cusp (Haerendel et al., 1978), but how their structure changes as they propagate is so far unknown. (vi) What is their signature in the ionosphere? Concurrent observations of FTEs and ionospheric flow bursts have been made (see, for example Wild et al., 2001), but a coherent picture has still to emerge. (vii) How does the semiannual reconnection rate vary quantitatively? Such an estimate would allow to estimate the contribution of FTEs to the reduction of geomagnetic activity during solstice. These, and other questions, will be addressed in subsequent papers.

Acknowledgements. This work was supported by the National Science Foundation through grant ATM-0353211 and by the National Aeronautics and Space Administration through grant NAG5-12107. Computations were performed at the San Diego Supercomputer Center and at the National Center for Supercomputing Applications.

Topical Editor T. Pulkkinen thanks two referees for their help in evaluating this paper.

\section{References}

Axford, W. I. and Hines, C. O.: A Unifying Theory of High Latitude Geophysical Phenomena and Geomagnetic Storms, Can. J. Phys., 39, 1433-1464, 1961.

Berchem, J. and Russell, C. T.: Flux transfer events in the magnetopause: Spatial distribution and controlling factors, J. Geophys. Res., 89, 6689-6703, 1984.

Cliver, E. W., Kamide, Y., and Ling, A. G.: Mountains versus valleys: Semiannual variation of geomagnetic activity, J. Geophys. Res., 105, 2413-2424, 2000.

Cowley, S. W. H.: Plasma Poulations in a Simple Open Model Magnetosphere, Space Sci. Rev., 26, 217-275, 1980.

Cowley, S. W. H.: The causes of convection in the Earth's magnetosphere: A review of developments during the IMS, Rev. Geophys. Space Phys., 20, 531-565, 1982.

Elphic, R. C.: Observations of Flux Transfer Events: A Review, in: Physics of the Magnetopause, edited by:. Song, P., Sonnerup, B. U. Ö., and Thomsen, M. F., vol. 90, AGU Monogr. Ser., American Geophysical Union, 225-233, 1995.

Farrugia, C. J., Rijnbeek, R. P., Saunders, M. A., Southwood, D. J., Rodgers, D. J., Smith, M. F., Chaloner, C. P., Hall, D. S., Christiansen, P. J., and Wooliscroft, L. J. C.: A multi-instrument study of flux transfer event structure, J. Geophys. Res., 93, 14465$14477,1988$.

Fedder, J. A., Slinker, S. P., Lyon, J. G., and Russell, C. T.: Flux transfer events in global numerical simulations of the magnetosphere, J. Geophys. Res., 107, 1048, doi:10.1029/2001JA000 025, 2002.
Fu, Z. F. and Lee, L. C.: Simulation of multiple X-line reconnection at the dayside magnetopause, Geophys. Res. Lett., 12, 291-294, 1985.

Haerendel, G., Paschmann, G., Sckopke, N., Rosenbauer, H., and Hedgecock, P. C.: The frontside boundary layer of the magnetosphere and the problem of reconnection, J. Geophys. Res., 83, 3195-3216, 1978.

Hakkinen, L. V. T., Pulkkinen, T. I., Pirjola, R. J., Nevanlinna, H., Tanskanen, E. I., and Turner, N. E.: Seasonal and diurnal variation of geomagnetic activity: Revised Dst versus external drivers, J. Geophys. Res., 108, 2, doi:1029/2002JA009428, 2003.

Harten, A.: High resolution schemes for hyperbolic conservation laws, J. Comput. Phys., 49, 357-393, 1983.

Harten, A. and Zwas, G.: Self-adjusting Hybrid Schemes For Shock Computations, J. Comput. Phys., 9, 568-583, 1972.

Kawano, H., Kokubun, S., and Takahashi, K.: Survey of transient magnetic field events in the dayside magnetosphere, J. Geophys. Res., 97, 10 677-10 692, 1992.

Klumpar, D. M., Fuselier, S. A., and Shelly, E. G.: Ion composition measurements within magnetospheric flux transfer events, Geophys. Res. Lett., 17, 2305-2308, 1990.

$\mathrm{Ku}$, H. C. and Sibeck, D. G.: Flux transfer events produced by bursty merging at a single X line, J. Geophys. Res., 103, 14 965$14978,1998$.

Ku, H. C. and Sibeck, D. G.: Flux transfer events produced by the onset of merging at multiple X lines, J. Geophys. Res., 105, 2657-2675, 2000.

Le, G., Russell, C. T., and Kuo, H.: Flux transfer events: Spontaneous or driven?, Geophys. Res. Lett., 20, 791-794, 1993.

Lee, L. C. and Fu, Z. F.: A theory of magnetic flux transfer at the Earth's magnetopause, Geophys. Res. Lett., 12, 105-108, 1985.

Lee, L. C. and Fu, Z. F.: Multiple X Line Reconnection, 1. A Criterion for the Transition From a Single X Line to a Multiple X Line Reconnection, J. Geophys. Res., 91, 6807-6815, 1986.

Liu, Z. X. and Fu, Y. D.: Local magnetic reconnection caused by vortices in the flow field, Geophys. Res. Lett., 15, 1752-1575, 1988.

Lockwood, M. and Wild, M. N.: On the quasi-periodic nature of magnetopause flux transfer events, J. Geophys. Res., 98, 59355940, 1993.

Ogino, T., Walker, R. J., and Ashour-Abdalla, M.: A magnetohydrodynamic simulation of the formation of magnetic flux tubes at the Earth's dayside magnetopause, Geophys. Res. Lett., 16, 155-158, 1989.

Paschmann, G., Sonnerup, B. U. Ö., Papamastorakis, I., Sckopke, N., Haerendel, G., Bame, S. J., Asbridge, J. R., Gosling, J. T., Russell, C. T., and Elphic, R. C.: Plasma acceleration at the Earth's magnetopause: Evidence for reconnection, Nature, 282, 243-246, 1979.

Paschmann, G., Haerendel, G., Papamastorakis, I., Sckopke, N., Bame, S. J., Gosling, J. T., and Russell, C. T.: Plasma and magnetic field characteristics of magnetic flux transfer events, J. Geophys. Res., 87, 2159-2168, 1982.

$\mathrm{Pu}, \mathrm{Z}$. Y., Yei, M., and Liu, Z. X.: Generation of vortex-induced tearing mode instability at the magnetopause, J. Geophys. Res., 95, 10 559-10 566, 1990.

Raeder, J.: Modeling the magnetosphere for northward interplanetary magnetic field: Effects of electrical resistivity, J. Geophys. Res., 104, 17 357-17 367, 1999.

Raeder, J.: Global Magnetohydrodynamics - A Tutorial, in: Space Plasma Simulation, edited by: Büchner, J., Dum, C. T., and Scholer, M., Springer Verlag, Berlin Heidelberg New York, 2003. 
Raeder, J., Berchem, J., and Ashour-Abdalla, M.: The Geospace Environment Modeling Grand Challenge: Results from a Global Geospace Circulation Model, J. Geophys. Res., 103, 1478714 797, 1998.

Raeder, J., McPherron, R. L., Frank, L. A., Paterson, W. R., Sigwarth, J. B., Lu, G., Singer, H. J., Kokubun, S., Mukai, T., and Slavin, J. A.: Global simulation of the Geospace environment modeling substorm challenge event, J. Geophys. Res., 106, 381395, 2001a.

Raeder, J., Wang, Y. L., and Fuller-Rowell, T.: Geomagnetic storm simulation with a coupled magnetosphere - ionosphere - thermosphere model, in: Space Weather, AGU Geophys. Monogr. Ser., edited by: Song, P., Siscoe, G., and Singer, H. J., vol. 125, American Geophysical Union, 377-384, 2001b.

Rijnbeek, R. P., Cowley, S. W. H., Southwood, D. J., and Russell, C. T.: Observations of reverse polarity flux transfer events at the Earth's magnetopause, Nature, 300, 23-26, 1982.

Rijnbeek, R. P., Cowley, S. W. H., Southwood, D. J., and Russell, C. T.: A survey of dayside flux transfer events observed by ISEE 1 and 2 magnetometers, J. Geophys. Res., 89, 786-800, 1984.

Russell, C. T. and Elphic, R. C.: Initial ISEE Magnetometer Results: Magnetopause Observations, Space Sci. Rev., 22, 681$715,1978$.

Russell, C. T. and Elphic, R. C.: ISEE Observations of Flux Transfer Events at the Dayside Magnetopause, Geophys. Res. Lett., 6, 33-36, 1979.

Russell, C. T. and McPherron, R. L.: Semiannual variation of geomagnetic activity, J. Geophys. Res., 78, 92-108, 1973.

Sato, T., Shimada, M., Hayashi, T., and Watanabe, K.: Formation of field-twisting flux tubes on the magnetopause and solar wind particle entry into the magnetosphere, Geophys. Res. Lett., 13, 801-804, 1986.

Saunders, M. A., Russell, C. T., and Sckopke, N.: Flux transfer events: Scale size and interior structure, Geophys. Res. Lett., 11, 131-134, 1984.

Scholer, M.: Magnetic flux transfer at the magnetopause based on single X line bursty reconnection, Geophys. Res. Lett., 15, 291294, 1988.

Scholer, M.: Undriven magnetic reconnection in an isolated current sheet, J. Geophys. Res., 94, 8805-8812, 1989.

Scholer, M.: Models of Flux Transfer Events, in: Physics of the Magnetopause, edited by: Song, P., Sonnerup, B. U. Ö., and Thomsen,M. F., vol. 90, AGU Monogr. Ser., American Geophysical Union, 235-245, 1995.
Shi, Y., Wu, C. C., and Lee, L. C.: A study of multiple X line reconnection at the dayside magnetopause, Geophys. Res. Lett., 15, 295-298, 1988.

Shi, Y., Wu, C. C., and Lee, L. C.: Magnetic field reconnection patterns at the dayside magnetopause: An MHD simulation study, J. Geophys. Res., 96, 17 627-17 650, 1991.

Siscoe, G. L., Erickson, G. M., Sonnerup, B. U. Ö., Maynard, N., Schoendorf, J. A., Siebert, K. D., Weimer, D. R., White, W. W., and Wilson, G. R.: Flow-through magnetic reconnection, Geophys. Res. Lett., 29(13), 4-1, 2002.

Sonnerup, B. U. Ö.: On stress balance in flux transfer events, J. Geophys. Res., 92, 8613, 1987.

Southwood, D. J., Saunders, M. A., Dunlop, M. W., MierJedrzejowicz, W. A. C., and Rijnbeek, R. P.: A Survey of Flux Transfer Events Recorded by the UKS Spacecraft Magnetometer, Planet. Space Sci., 34, 1349-1953, 1986.

Thomsen, M. F., Stransberry, J. A., Bame, S. J., Fuselier, S. A., and Gosling, J. T.: Ion and electron velocity distributions within flux transfer events, J. Geophys. Res., 92, 12 127-12 136, 1987.

Van Leer, B.: Towards the ultimate conservative difference scheme, I. The quest for monotonicity, in: Lecture Notes in Physics, vol. 18, Springer Verlag, Berlin, 163, 1973.

Van Leer, B.: Towards the ultimate conservative difference scheme, II. Monotonicity and conservation combined in a second order scheme, J. Comp. Phys., 14, 361-370, 1974.

Van Leer, B.: Towards the ultimate conservative difference scheme, III. Upstream centered finite difference schemes for ideal compressible flow, J. Comp. Phys., 23, 263-275, 1977.

Wang, Y. L., Raeder, J., Russell, C. T., Phan, T. D., and Manapat, M.: Plasma depletion layer: Event studies with a global model, J. Geophys. Res., 108, 1010, doi:10.1029/2002JA009281, 2003.

Wild, J. A., Cowley, S. W. H., Davies, J. A., Khan, H., Milan, S. E., Provan, G., Yeomans, T. K., Balogh, A., Dunlop, M. W., Fornacon, K.-H., and Georgescu, E.: First simultaneous observations of flux transfer events at the high-latitude magnetopause by the Cluster spacecraft and pulsed radar signatures in the conjugate ionopsphere by the CUTLASS and EISCAT radars, Ann. Geophys., 19, 1491-1508, 2001,

SRef-ID: 1432-0576/ag/2001-19-1491. 